\title{
Study on Causes of Disappearance of Wax Printing Technique in the Central Plains Area during Song Dynasty
}

\author{
Bin LI ${ }^{1 \mathrm{~A}}$, Hong-Guang YE ${ }^{1 \mathrm{~B}}$, An-Ding LIU ${ }^{2 \mathrm{C}}$ and Xiao-Jing FANG ${ }^{1 \mathrm{D},{ }^{*}}$ \\ ${ }^{1}$ College of Fashion, Wuhan Textile University, Wuhan, 430073, China \\ Library, Wuhan Textile University, Wuhan, 430073, China \\ alibin790121@qq.com, b836001463 @qq.com,

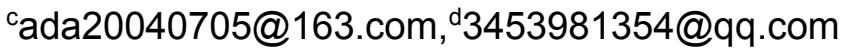

Keywords: Wax Printing, Disappearance, Song Dynasty, the Central Plains Area.

\begin{abstract}
The aim of this paper is to analyze the sudden disappearance of the wax printing technique in the Central Plains area during Song dynasty. We analyzed historical literatures, textile archaeology, and various other methods to summarize the main reasons leading to the disappearance of the wax printing from three aspects: political, economic, and technical factors. Political factors are reflected in the promulgation and implementation of dyeing injunction in the Song Dynasty; the government did not support this technique in folklore for the process wasted a lot of resources. Economic factors are reflected in the difficulty to obtain a large number of beeswax, the raw material for wax printing, because of the reduced territory in Song Dynasty. Technical factors are reflected in the generation and widespread of the new alkaline printing process, which accelerated the demise of wax printing.
\end{abstract}

\section{Introduction}

Wax printing, also known as "Laxie", was one of the four distinctive printing techniques in ancient China; the other three were Jiaxie, Zaxie and Huixie. The principle for wax printing is very simple, namely using wax as the resist agent due to its thermal performance and resist dyeing ability. After coating the high temperature liquid wax onto the fabric to form a pattern, the wax will be solidified to form a water resistant layer when the temperature goes back to normal; then put the fabric into dye liquor with repeatedly dip-dye, and wash the fabric in order to remove all the wax. Even though the origin of wax printing in the Central Plains area was unable to be confirmed, this technique has already been developed quite maturely in the Tang dynasty, which has not only the hand drawing and screen printing method, but also the monochromatic and polychromatic patterns [1]. However, the wax printing in Central Plains area suddenly disappeared in Song dynasty. This paper summarized the main reasons which are: (1) dyeing prohibition was an important political factor that deteriorated the development environment of wax printing; (2) the lack of raw materials was an important economic factor that influenced the development of wax printing; (3) the generation of alkaline printing was an important technical factor that accelerated the demise of wax printing in the Central Plains area.

\section{Discussions and Analyses}

From the Sui and Tang Dynasties to the Southern Song Dynasty Was the Period When the Flourishing Wax Printing Technique in the Central Plains Area Withered Away

The origin of China's wax printing technique has always been a controversial topic in the textile history field. Mr. Chen Weiji believed that China's wax printing technique can be traced back to the minority nationalities in the southwest of the Qin and Han dynasties. At that time, the southwest minorities have begun to use the waterproof properties of beeswax, insect wax and resin material for fabric dyeing. With the development of "Tu-gong" system and the exchanges of culture and trade, the wax printing technique gradually spread to the Central Plains area [2]. Mr. Zhao Feng had researched on the unearthed wax printing products of a half-naked goddess in Xinjiang Niya. Based on the India 
Gandhara culture factors which showed on both the goddess's hand-held cornucopias as well as the lights behind her, Zhao Feng concluded that China's wax printing technique was originated from India. Regardless of whether the origin was from China's ethnic areas or foreign areas, the main problem that needs to be solved is when exactly was this technique introduced into the Central Plains region and how was it developed.

\section{Wax printing obtained rapid development in the Central Plains area in Tang Dynasty}

In 1959, two wax printing fabrics of the Northern dynasties (396-581) was unearthed from Wuyu-laike ancient city site in Yutian, which was a blue wool fabric and a blue cotton cloth; but these two pieces of cultural relics were not able to prove that the wax printing technique already existed in the Central Plains region in Northern Dynasties. As commonly known, wool fabric was the main fabric of people in the minority areas of northern China. If a wool fabric existed in Central Plains region, it was usually introduced into the Central Plains area by the minority areas. Besides, cotton fabric was also used as tribute from the northwest and southwest minority because fabrics that used in the Central Plains were mainly made from silk, Ge and hemp. Therefore, these two pieces from Northern Dynasties must not be used as evidence to support that wax printing technique existed in Central Plains area.

Nonetheless, a couple of evidence has shown that the wax printing technique became popular in the Central Plains region during the Tang Dynasty.

Evidence 1: In 1968, a number of residual streamers of Tang Dynasty were discovered in Dunhuang Mogao Grottoes, which included some wax printing fabrics. These were silk with cloud and birds flowers pattern in blue background, silk with ganoderma and plants pattern in red background, silk with cloud and birds flowers pattern in yellow background as well as silk with plants pattern in brown background. It can be assumed from these fabrics that silk had become popular for wax printing at that time, since the blank for all these fabrics were silk; but this could only proved the possibility that these fabrics were from the Central Plains area, because GaoChang Kingdom in Western Regions also mastered the silk knitting techniques. However, since ganoderma has been widely used as auspicious sign in fabric patterns by the Han nationality while other nations did not, it can be proved that the production process was in the Central Plains region. According to points mentioned above, we concluded that the wax printing techniques in the Central Plains area was rooted and developed at least during the Tang Dynasty.

Evidence 2: The wax printing fabrics from Tang dynasty which is now stored in a museum called Nara Shosoin in Japan. Currently, a large quantity of wax printing products is stored in Nara Shosoin in Japan. For example, the tree with elephant and sheep wax printing screen (Fig.1); it was made from furs and dyed with gorgeous pattern with wax printing method [3]. The figure was designed through fine process which made the screen with rich levels and elegant appearance. According to Japanese literature document, the "screen" of Tang Dynasty was a famous decorative articles and was exported to foreign countries as a precious gift of friendship [4]. Regardless of how it was introduced into Japan, the fact that these screen were produced in the Central Plains of Tang Dynasty was affirmed by many scholars both domestic and abroad.

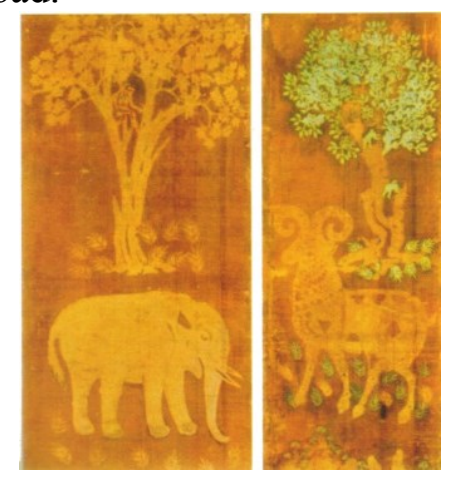

Fig.1. The tree with elephant and sheep wax printing screen 
In respect of the previously mentioned evidence, the technique had long been out of its embryonic stage of development and entered into a more mature stage; the fluency pattern, clear edge, prominent topic and exquisite quality made it possible to be given to foreign countries as an official gift or carry out for foreign trade.

\section{Wax Printing Has Rapidly Disappeared in the Central Plains Area during Southern Song Dynasty}

Wax printing did reach a high level in Tang dynasty, however, it was rarely known in the Central Plains area during Southern Song Dynasty. This technique was once recorded in Linwaidaida of Southern Song scholar Zhou Qufei (1135 1189) that: "Yao people dyed blue cloth with patterns. They placed cloth between two pieces of carved woods, poured the liquid wax through the hollowing patterns; then released the board and take out the cloth to place it into blue dyestuff. After that, placed the stained blue cloth in boiling water to remove the wax. This method formed the impressive wax-breaking pattern, which only Yao people could achieve." [5]. Thus, we can infer that the wax printing technique had disappeared in the Han nationality living area during the Southern Song Dynasty, otherwise Zhou Qufei would not be able to record the wax printing process of Yao people as an anecdote.

The space-time transformation of ancient wax printing process caused the loss of the development of this technique in the Central Plains area and only slowly developed in the Southwest minority areas. Presently, when it comes to wax printing, people usually regard it as a dyeing process of the ethnic minorities in Southwest China, while its unsurpassable refulgence in the Central Plains area of Tang Dynasty is ignored.

\section{Reasons for Wax Printing Disappeared in the Central Plains}

China's textile historians generally believe that the reason for the disappearance of wax printing in the Central Plains was mainly caused by Song dynasty's prohibition on dyeing. Based on the analysis of the historical data of the Tang and Song Dynasties, we suggested that the Song dynasty's prohibition had a bad effect in the development process of wax printing in the Central Plains region, but it was not the main reason. The reason for its disappearance should be caused by many factors, such as politics, economy, technology and so on. In general, Song's prohibition on dyeing was the political reason, while the lack of raw materials was an important economic factor, and the appearance of alkaline printing was the key technical factor for the disappearance of wax printing in central plains.

\section{Song Dynasty's Prohibition on Dyeing Was the Political Reason}

Textile historians generally agree that the Song dynasty's prohibition on dyeing was due to the prevalence of dyeing, causing a large amount of manpower and material resources to be used to make exquisite dyeing clothing and daily necessities. In order to curb extravagance and advocate simplicity, the government imposed a ban on it. However, the real situation may not be the case. If the purpose was to advocate simplicity, the more complex tapestry and brocade needed to be banned, but they developed rapidly in the Song dynasty instead. We believe that the reason was the close relationship between dyeing and the "Yu-fu" system. According to the History of Song annals on Yu-fu system, the imperial edicts by Emperor Huizong (1082-1135, 1100-1126) of Song in $2^{\text {nd }}$ year of Zheng He (1112): "Valerian silk made in the palace for army to guard and to distinguish the treacherous, while civil production was banned". It was not difficult to see that the dyeing prohibition was for distinguishing the uniform between army and civilians. However, even though the purpose was different, they still had same effect which heavily hit all kinds of dyeing and printing industry including wax printing.

\section{Lack of Raw Materials Was an Important Economic Factor}

Beeswax was the key raw material for wax printing in the Central Plains area, while it was used not only for wax printing but for other fields as well. In fact, beeswax was used for lighting in as early as Han dynasty. According to A Miscellany of the Western Capital by Ge Hong of Jin dynasty: "King of 
Nanyue Kingdom dedicated five bottles of Honey and two hundred beeswax to Gao emperor". Besides, a mural with candle was found in the tomb of Princess Yongtai of Tang in Qianxian, Shanxi. [6]. In addition to making candles, beeswax also had following purposes. Firstly, it was used in medicine. Beeswax was a traditional drug in ancient times, used for curing diarrhea and relieving pains. For example, Three Yellow Jewels Wax Pill was for the treatment of traumatic injury with excellent results. Secondly, it was used as the seal of letters and official documents to prevent information leakage. In Song dynasty, wax pill letters were used for transmission of information during the war, and was even used as the communication way as well as war and peace declaration with Liao and Jin. Thirdly, beeswax was an important auxiliary material for statue sculpturing and book framing. Lastly, Beeswax as an anti-corrosion and lubrication material, was widely used in footwear, cars, textile and other industries in ancient times [7]. Therefore, beeswax was widely utilized in the Central Plains region in ancient times.

However, beekeeping industry in Central Plains could not provide enough beeswax to meet production and life needs. According to Song dynasty's documents, the tribute of beeswax from various regions were: One hundred pounds of beeswax from Luoyang, one hundred pieces of beeswax from Dengzhou, thirty pounds of beeswax from Xi'an, ten pounds of beeswax from Pengshui, etc. [8] It was not difficult to find that the beeswax quantity which tribute in Central Plains was relatively small, at most one hundred pounds and sometimes only ten pounds. In addition, one catty during Song dynasty was equivalent to the current six hundred and forty grams, therefore, beeswax was expensive during that time.

There is a paradox: The wax printing technique gradually disappeared in the Song dynasty, but how did it develop so well in the Tang dynasty while its beekeeping industry was far weaker? First of all, we suggest that the emergence of this phenomenon was closely related to the rule area of these two dynasties. It was commonly known that Tang had a vast territory, which meant it had more regions to get beeswax. According to New History of the Tang Dynasty, Today's Shanxi, Anhui, Hubei, Sichuan, Gansu, Zhejiang, Fujian and Guizhou all made beeswax tribute. [9]In addition, Tang accelerated the control of the southwestern region. In Jiannan Dao(known today as Western Sichuan and most parts of Yunnan), Jiangnan Dao (known today as Western Hunan, Guizhou and Northern Guangxi), Lingnan Dao(known today as most parts of Guangxi, Eastern Yunnan, South Indochina), a total of 415 ethnic minority counties were set up. The southwest frontier of the Tang Dynasty developed rapidly and imported large number of local products into the mainland, which were mainly medicinal products, handicrafts, rare animals and plants and their products. These frontiers contained Guizhou area where had the most beeswax tribute.[10][11] On the contrary, the boundaries of the Song dynasty were greatly reduced, lost not only the sixteen prefectures but also the control of few beeswax production areas in Southwest minority region. Therefore, the number of beeswax in the Central Plains region might be much lower than that of the Tang Dynasty.

Secondly, because of the superior trading environment in Tang Dynasty, it was inevitable that the external input conditions of beeswax were much better when compared with the Song dynasty. During Tang Taizong period, the western region was under its control while the Silk Road in the southwest and sea was opened, a large number of foreign goods swarmed into China. Even though there was no clear historical record on beeswax from external import, but we could not deny the possibility. On the contrary, condition was not optimistic in Song dynasty. The northwest passage was blocked by Xixia as well as the control for the Southwest was lost, only the sea route was able for trading. Therefore, external ways for obtaining beeswax were much less than that in the Tang Dynasty, which blocked the development of wax printing.

\section{Alkaline Printing Was the Important Technical Factor}

Alkaline printing had incomparable advantages compared to wax printing. First, from the view of resist-dyeing agent for alkaline printing, it was basically the vegetal powder including soybean powder and mung bean powder, while the auxiliary materials were the most common lime powder. Therefore, the sizing agent was much cheaper than that of wax printing and was easy to obtain. 
Second, technically the process level was not high for alkaline printing since it did not require heating. On the contrary, wax printing required heating process because of its rapid solidification performance, resulted in the demands for higher skilled craftsmen. Finally, from the view of product standardization, alkaline printing could achieve it easily while wax printing could not. Because of the advantage of no requirement for heating process, the quality control for hollow paper can already maintain the alkaline printing with a high degree of consistency. Based on above three points, there was no doubt for wax printing to gradually disappear in the civil society after the appearance of alkaline printing.

The disappearance of wax printing in Central Plains region did not mean its extinction. There was always room for it as long as it could be loved by the upper class, since the royal palace would not consider the production cost of wax printing. However, it was unfortunately that the Yuan Dynasty, which was founded by the northern nomadic Mongols, replaced the Song dynasty. The cruel rule and national discrimination of the leaders made southern economy and culture suffered heavy losses, and dyeing industry suddenly depressed. More importantly, the warlike habits, the pursuit of luxury lifestyle as well as the ruler's respect for the royalty and nobility culture of West Asia, increased their fondness of rich and magnificent brocade in the Yuan Dynasty, while all kinds of pure elegant dyeing could not. Thus, the once popularized wax printing technique disappeared in the Central Plains area, moved southward and achieved eternity prosperous in the southwest region.

\section{Conclusion}

By analyzing the reasons of wax printing disappearance in the Central Plains region, there was no express prohibition of wax printing in the Song Dynasty. However, it caused the downturn of the whole dyeing industry. Inevitably, this must have an influence on the application of wax printing in civilian stratum in central plain. Indeed, the price of beeswax was the most important reason for the restriction of wax printing development in the Central Plains. When the cheaper alkaline printing had appeared, it was inevitable for wax printing to be placed in the civilian stratum in Central Plains.

\section{References}

[1] Jin Hu, Xiao-Ya Jian. Research on Anatkina Cloth and Wax Printing. Guizhou Culture and History, 2(2001)41-45.

[2] Wei-Ji Cheng. History of Textile Technology of Ancient China, Beijing, 1984.

[3] Ying-zhao Bao. Brief Discussion on Colored Wax Printing. Printing and Dyeing, 3(1985)25-26.

[4] Ran-Ren MingShi. Study on the History of Weaving Dye Making, Tokyo, 1927.

[5] Xiong-lue Yu. Blue Calico of China. Beijing, 2008.

[6] Shu-Pei Yang. The Understanding of Bees and the Beekeeping Technology in Ancient China. Agricultural Archaeology, 1(1988)242-251.

[7] Feng-Chen Ge, Dong-Hai Chen, Yan-Fang Li. The Antiquity Culture of Changbai Bee (nineteen) -Research on Ancient Beeswax, Apiculture of China, 1(1998)27-28.

[8] Xian-Yun Zhang. Study on Apiculture of the Sang Dynasty. Journal of Bee, 5(2007)14-16.

[9] Rui Guo. Preliminary Study on Apiculture of the Tang Dynasty. Research on Chinese Social Economic History, 1(2011)5-10.

[10] Tie Fang. The Ruling Ideology of the Tang Dynasty Rulers and the Countermeasures to the Southwest Frontier. Journal of Yunnan Institute of Nationalities (philosophy and social sciences edition), 2(2001)51-54.

[11] Chen He, Wen-Bin Yang. The Wax Printing of Gui Zhou. Suzhou, 2009. 